\title{
Los factores relacionados con la calidad de vida de los adultos mayores institucionalizados de Bucaramanga
}

\author{
Factors related to the quality of life of institutionalized elderly in Bucaramanga
}

\author{
Sonia Constanza Concha Sánchez
}

Para citar este artículo: Concha - Sánchez SC. Los factores relacionados con la calidad de vida de los adultos mayores institucionalizados de Bucaramanga. UstaSalud. 2017;16: 7-16

Licencia Creative Commons \begin{abstract}
(c) (1) (\$) lo tanto, los lectores pueden acceder libremente a los artículos en su formato .pdf, igualmente podrán descargarlos y difundirlos; sin embargo no podrán modificarlos o alterarlos, adicionalmente se debe reconocer la autoría de las personas que figuran en las publicaciones, pero estas no podrán comercializadas.
\end{abstract}

\section{RESUMEN}

Objetivo: Identificar los factores relacionados con la calidad de vida en los adultos mayores de seis instituciones geriátricas de Bucaramanga.

Materiales y métodos: Un estudio observacional analítico de corte transversal se realizó sobre 221 adultos mayores vinculados a seis hogares geriátricos del área metropolitana de Bucaramanga, en quienes se recolectaron variables sociodemográficas, condición sistémica, CV, condición oral, capacidad física funcional, salud mental y soportes sociales. Con los datos recolectados se calcularon medidas de resumen, según la naturaleza de las variables, se efectúo un análisis de factores para definir la variable condición oral. Para el bivariado se relacionó calidad de vida con cada variable en estudio, mediante test de $\mathrm{Chi}^{2}$ o exacto de Fisher. Se aplicó análisis de regresión logística para el cálculo de los OR con sus respectivos IC95\%, métodos de corrección de los OR y se evalúo la bondad de ajuste del modelo.

Resultados: Estar institucionalizado, no realizar labores y estar deprimido (todas con RR 1,6) se asociaron con una mala calidad de vida en las personas vinculadas a los seis hogares geriátricos analizados.

Conclusiones: La depresión, la institucionalización y no realizar actividades son factores que parecen comprometer la calidad de vida de ancianos residentes en estas instituciones geriátricas.

Palabras clave: Adulto mayor, calidad de vida, depresión.

\section{ABSTRACT}

Objective: To determine the relationship between mental health and the quality of life (QL) in the institutionalized elderly

Materials and methods: An analytical cross section observational study was made in 221 elderly, who lived in six Bucaramanga elderly institutions, who met the eligibility criteria. The variables of the study were social-demographic; systemic condition, the QL, the oral condition (OC), the functional physical capacity, mental health, and the social supports. In the analysis, summary statistics were calculated according to the type of each variable. Analysis of factors was made to determine the attributes that integrated OC. The dependent variable (QL) was related to each other variables by $\mathrm{Chi}^{2}$ and Fisher's exact test, the crude OR and intervals of confidence were calculated. Logistic regression analysis and methods of correction of the OR were applied, the RR were obtained and the fit goodness of the model was also evaluated.

Results: To be institutionalized, not to work, and to be depressed (RR 1.6) were associated with a bad quality of life in the evaluated institutional elderly.

Conclusions: The depression, the institutionalization and the fact of not executing activities are related factors to the quality of life in this city institutionalized elderly.

Keywords: Aged, quality of life, depression.
Grupo de Investigación Salud Integral Bucal, Facultad de Odontología, Universidad Santo Tomás, Bucaramanga, Colombia.

Autor de correspondencia: Sonia Constanza Concha Sánchez Correo electrónico: sonia.concha@ustabuca.edu.co
Recibido para publicación: 22 de febrero de 2017. Aceptado para publicación 5 de mayo de 2017. 


\section{INTRODUCCIÓN}

El envejecimiento poblacional genera profundos cambios en todos los aspectos de la vida de las personas, de la sociedad en general y en el ejercicio de los profesionales de todas las áreas, que deben afrontar este reto ofreciendo alternativas pertinentes y oportunas frente a las necesidades de los individuos y las colectividades. Los países latinoamericanos enfrentan el reto de envejecer en medio de las dificultades económicas, sociales y políticas que registran los países de la región y de las profundas desigualdades que existen entre los diferentes grupos poblacionales ${ }^{1,2}$.

Colombia en particular enfrenta el reto simultáneo relacionado con el desarrollo, el envejecimiento, el desplazamiento forzado y la violencia, la reducción en las tasas de natalidad y el incremento en el número de mujeres mayores frágiles, las profundas brechas socioeconómicas y las grandes inequidades por región geográfi$\mathrm{ca}$, entre otras ${ }^{2,3,4}$ situaciones que se deben analizar, con el ánimo de ofrecer respuestas y mejores alternativas de vida y de salud a las personas mayores.

Si bien es cierto, el compromiso de lograr el bienestar de los adultos mayores es de todos, los profesionales de la salud son quizás los principales responsables de ofrecer no solo cantidad sino también calidad de vida $^{5}$; pero para poder responder a este reto es necesario comprender el impacto que tienen las estructuras sociales sobre los individuos; también, los cambios que ocurren durante el ciclo vital, la variabilidad entre los individuos, los cambios asincrónicos que acontecen en un mismo individuo a nivel de las diferentes estructuras y la necesidad de estudiar todo lo relacionado con el envejecimiento normal y patológico ${ }^{6,7}$.

Vivir bien es un concepto que puede tener diferentes significados para cada persona, para un grupo social y para una generación en particular; a pesar de ello, existen ciertos criterios en los que parece hay acuerdo cuando se habla de calidad de vida, dentro de los que cabría considerar en primera instancia tener la capacidad y la oportunidad de determinar la propia vida, que se alcanza con mayor facilidad cuando se cuenta con educación, salud y bienes materiales. En segundo lugar, el considerarse una persona valiosa es otro factor que incentiva la calidad de vida, que se fortalece con las redes sociales que se establecen con la familia y la co- munidad. Otro criterio que incide sobre la posibilidad de vivir bien es la posibilidad de disfrutar de condiciones seguras para vivir, de libertades tanto civiles como políticas y la posibilidad de realizarse como un sujeto político, con deberes, pero también derechos como ciudadano. Finalmente, unas condiciones ambientales adecuadas que promuevan el desarrollo integral de los individuos y de los colectivos ${ }^{1,8}$. Con base en estos criterios se puede afirmar que la calidad de vida es una construcción compleja, multifactorial que se mide a través de diferentes indicadores; sin embargo, la percepción subjetiva también es un criterio importante a la hora de evaluar el buen vivir ${ }^{1,9}$.

En el marco del buen vivir existe el concepto de calidad de vida relacionada con salud (CVRS). La CVRS cuenta con múltiples definiciones; con este fundamento se han elaborado modelos multifactoriales, como un mecanismo para cuantificar las consecuencias de la enfermedad y los tratamientos sobre la calidad de vida y la salud, soportada en la posibilidad que tienen los individuos para vivir plenamente. La CVRS está integrada por cuatro componentes fundamentales: el estado físico, la condición laboral y el estado funcional; la salud mental; las redes sociales, y las sensaciones somáticas ${ }^{1,10}$.

En el campo de la gerontología la comprensión de la calidad de vida en el adulto mayor permite analizar, además de los aspectos biológicos, las condiciones de vida, la capacidad para desarrollar actividades de la vida cotidiana que promueven en el adulto mayor el buen vivir, y su independencia; aspectos que se deben ajustar con base en la esperanza de vida y en las expectativas que el adulto mayor tiene frente a la vida que desea vivir. Los datos epidemiológicos en todas las regiones del mundo muestran cómo la enfermedad y la discapacidad aumentan con la edad; frente a esta realidad epidemiológica se evidencia la necesidad de generar nuevas concepciones relacionadas con la salud en la edad avanzada, que promuevan la independencia y destaquen sus valiosos aportes a la sociedad; por lo que conceptos como los de mortalidad, morbilidad y el riesgo no logran capturar la compleja realidad que enfrentan las personas mayores; los abordajes orientados promover la autonomía enriquecen el concepto de calidad de vida relacionada con la salud en el adulto mayor ${ }^{1,11}$. En este sentido, el ingreso de los adultos mayores a instituciones geriátricas representa grandes problemas tanto para ellos como para las personas que los atienden, pues la institucionalización puede deteriorar la calidad de vida 
del adulto mayor, comprometer su condición física, sus relaciones sociales y su salud mental.

La OMS define la salud mental como el bienestar que experimentan las personas como resultado de su buen funcionamiento, tanto en el nivel cognitivo, afectivo como en el conductual; así como en el despliegue óptimo de sus potencialidades individuales para la convivencia, el trabajo y la recreación ${ }^{12}$. En términos generales, cualquier enfermedad afecta la calidad de vida, pero los problemas mentales, incluyendo los desórdenes de ansiedad, los depresivos y los afectivos deterioran aún más la calidad de vida de las personas mayores ${ }^{13}$.

En el 2000 había en el mundo más de 600 millones de personas mayores de 60 años, se espera que para el 2020 esta cifra aumente en un $70 \%$. Este rápido aumento de la población anciana trae consigo problemas físicos y mentales relacionados con la edad, incluyendo un mayor riesgo a demencia, reducción del bienestar general y de la calidad de vida, por lo que la promoción de la salud mental es un asunto que le atañe a los sectores gubernamentales, comerciales y de manera particular a los profesionales de la salud ${ }^{14}$.

Considerado lo expuesto, el objetivo de la presente investigación es identificar los factores relacionados con la calidad de vida (CV) en los adultos mayores de seis instituciones geriátricas de Bucaramanga.

\section{MÉTODOS}

Se realizó un estudio observacional analítico de corte transversal. La población de estudio estuvo conformada por la totalidad de adultos mayores vinculados a seis instituciones geriátricas de Bucaramanga y su área metropolitana. La muestra estuvo integrada por 221 adultos mayores. Como criterios de inclusión se consideraron ser adulto mayor vinculado a una de las instituciones involucradas en el estudio y se excluyeron las personas mayores con enfermedades severas, hipoacusia o trastornos siquiátricos mayores.

El tamaño de muestra se calculó mediante la rutina Statcal del paquete estadístico EPI-INFO 6.04, considerando los siguientes parámetros para un estudio de corte transversal: nivel de confianza del 95\%, poder del $80 \%$, relación expuesto no expuesto $1: 1$, prevalencia de mala calidad de vida en personas con patologías orales del $60 \%$ y en aquellos con una buena salud oral del $40 \%$; con R.R. 1,5 y OR 2,25 y una potencial atricción de la muestra de un 3\%. Los sujetos fueron elegidos mediante muestreo no probabilístico voluntario.

Para la realización de esta investigación se recolectaron las siguientes variables:

- Variable de salida o dependientes: calidad de vida, que se evalúo mediante Escala Análoga Visual con valores que oscilaron entre 0 y $100 \mathrm{~mm}$ y que se categorizó con base en la mediana, los valores cercanos a cero representaban una peor calidad de vida.

- Variables explicativas o independientes: en las que se incluyeron varios grupos de variables. El primero representado por la condición sistémica (enfermedades y medicación), experiencia de dolor (presencia, localización e intensidad), condición sensorial y del lenguaje soportado en el cuestionario general de salud (GHQ), apoyo social recolectado mediante el cuestionario de redes de apoyo y redes sociales de la vida adulta y la condición oral (derivada del análisis de factores caries dental, enfermedad periodontal, necesidad de prótesis, etc.). El segundo grupo que incluía la evaluación de la condición mental mediante el Minimental (Examen Mental Abreviado) y la depresión con base en el CES-D empleando los criterios establecidos para estos instrumentos cuando se aplican a personas mayores. En el tercer grupo se incluyó la variable orientada a evaluar la condición física, utilizando el ICF (Índice de Capacidad Funcional). En el último grupo se incluyeron las variables sociales y demográficas que se utilizaron en esta investigación y que fueron: escolaridad (total de años cursados por la persona mayor), género (hombre, mujer), tipo de afiliación al sistema de seguridad social (ninguno, subsidiado, contributivo y otro), ocupación o actividad que realiza dentro de la institución geriátrica (si hace o no hace actividades) y vinculación institucional (interno o externo).

Para recolectar cada una de las variables se hizo la estandarización de la examinadora en los criterios de 
diagnóstico de cada uno de los aspectos valorados, la realización de la prueba piloto en las que se verificaron procedimientos e instrumentos. La recolección de la información se realizó en cada una de las instituciones geriátricas. Antes de recolectar la información se revisó la historia clínica de cada adulto mayor, se determinó el cumplimiento de los criterios de inclusión, aspecto que ser verificó con el personal de enfermería o responsable de la atención en la institución. Se inició con la recolección de las variables sociodemográficas, posteriormente se aplicaron los cuestionarios relacionados con la salud general, funcionalidad, salud mental y de redes sociales y, finalmente, se efectuó el examen oral. La información recolectada se sistematizó por duplicado en Excel $^{\oplus}$ (Excel 2013; Microsoft Corporation, Redmont: WA, USA), se exportó a Epi Info $^{\text {tw }} 6.04$ (CDC, Atlanta, GA, USA, 2011) y mediante la rutina validate se verificó la calidad de la digitación y se hicieron los correctivos necesarios, finalmente se exportó y se procesó mediante el paquete estadístico Stata 9.0 (StataCorp, 2005. Stata Statistical Software: Release 9, College Station, TX: StataCorp LP.)

Para el análisis estadístico univariado se calcularon medidas de resumen. Frecuencias absolutas y relativas, se obtuvieron para las variables cualitativas y medidas de tendencia central (promedio, mediana), $y$ de dispersión (rango, varianza, desviación estándar e intervalos de confianza) para las cuantitativas. Se generó la variable salud oral desde un análisis de factores y a partir de eventos orales evaluados. Para el análisis bivariado se relacionó la variable de salida (calidad de vida categorizada) con todas las variables explicativas para esto se aplicaron test de Chi Cuadrado o test Exacto de Fisher. Posteriormente, se corrió un modelo logístico multivariado que relacionó la variable calidad de vida con las variables explicativas que registraron valores de $\mathrm{p}<0,20$; al modelo final se le aplicaron pruebas para valorar su bondad de ajuste; todo esto con base en lo referido por Greenland y Hosmer Lemeshow. Para finalizar se procedió a obtener las razones de prevalencia a partir de los Odd Ratio (OR) logrados del modelo de regresión logística, ajustado mediante el método de corrección de los OR definidos por Zhang y Yu ${ }^{15}$. Cabe señalar que para todo este análisis se consideró un nivel de significancia de $\mathrm{p} \leq 0,05$.

El presente estudio se acogió a la normatividad establecida en la Resolución 008430 de 1993 para inves- tigación en salud, la investigación se catalogó como de riesgo mínimo y en todo momento se respetaron los principios de autonomía, beneficencia, confidencialidad y privacidad de la información recolectada.

\section{RESULTADOS}

\section{Análisis univariado}

En las seis instituciones geriátricas se evaluaron 221 adultos mayores con una media de edad de 75 años y rango entre 50-96 años, una media de escolaridad de un año con rango entre 0 y 15 años y predominaron las mujeres (54,7\% (121)); el 81,5\% (180) de las personas mayores estaban internos en estas instituciones (Tabla 1).

Tabla 1. Descripción de las variables analizadas en los adultos mayores institucionalizados de Bucaramanga

\begin{tabular}{|c|c|c|}
\hline Variable & $\mathrm{N}^{\mathrm{o}}$ & $\%$ \\
\hline Total & 221 & \\
\hline Género Femenino & 121 & 54,7 \\
\hline \multicolumn{3}{|l|}{ Institución } \\
\hline Primera & 39 & 17,7 \\
\hline Segunda & 25 & 11,3 \\
\hline Tercera & 42 & 19 \\
\hline Cuarta & 68 & 30,8 \\
\hline Quinta & 11 & 5 \\
\hline Sexta & 36 & 16,3 \\
\hline Vinculación institucional interno & 180 & 81,5 \\
\hline Sin vinculación régimen de seguridad & 11 & 5 \\
\hline No realizan labores en institución & 78 & 35,3 \\
\hline Edad $>70$ años & 149 & 67,4 \\
\hline Escolaridad $\leq 1$ año & 118 & 53,4 \\
\hline Mala calidad de vida & 113 & 51,1 \\
\hline Registra dolor & 147 & 66,5 \\
\hline Enfermedades sistémicas $>2$ & 184 & 83,3 \\
\hline Medicación >1 & 106 & 48 \\
\hline Afección de órganos sentidos & 149 & 67,4 \\
\hline Redes de apoyo inadecuadas & 136 & 61,5 \\
\hline Redes sociales inadecuadas & 213 & 96,4 \\
\hline Depresión (CES-d) & 124 & 56,1 \\
\hline Deterioro mental (minimental) & 166 & 75,1 \\
\hline Capacidad física funcional deteriorada (ICF) & 126 & 57 \\
\hline Mala condición oral & 159 & 72 \\
\hline
\end{tabular}


Unas redes sociales inadecuadas en el 96,4\% (213), más de dos afecciones sistémicas en el 83,3\% (166), un estado mental deteriorado en el 75,1\% (166), una mala condición oral en el 72\% (159), compromiso en los órganos de los sentidos en un 67,4\% (149) y algún tipo de dolor en un 66,5\% (147) fueron las condiciones más prevalentes en estas personas mayores. (Tablas 1 y 2 ).

En cuanto a la calidad de vida, esta registró una media de $55,8 \pm 20,4$, con una mediana de 58 y un rango entre 12 y $100 \mathrm{~mm}$; al analizarla en forma categórica se observó que el 51,1\% (113) de las personas mayores evaluadas registró mala calidad de vida (Tabla 2).

\section{Análisis bivariado}

Las variables asociadas en forma estadísticamente significativas con la calidad de vida fueron: labor realizada, vinculación institucional, depresión y capacidad física funcional y se constituyeron en la base para la construcción del modelo logístico multivariado. Cada una de estas variables muestra una asociación positiva con una mala calidad de vida (Tabla 2).

\section{Análisis multivariado y ajuste de los OR}

El modelo multivariado de regresión logística ajustado permite evidenciar que permanecer interno en las instituciones geriátricas, no realizar labores y sufrir de depresión son aspectos que se asocian con una mala calidad de vida en las personas mayores vinculadas a las instituciones geriátricas estudiadas en Bucaramanga. Se evidencia el efecto de sobreestimación de los Odds Ratio en relación con los RR para este modelo (Tabla 3).

\section{Evaluación de bondad de ajuste del modelo}

Las pruebas de diagnóstico de la bondad de ajuste de la regresión para este modelo mostraron un $\mathrm{c}^{2}$ de Hosmer y Lemeshow de 7,98 ( $\mathrm{p}=0,4350)$ lo que indica un buen ajuste del modelo logístico. Así mismo, los residuales presentan un promedio y una varianza cercanos a cero y a uno respectivamente, apoyando los hallazgos anteriores. Complementariamente, el análisis gráfico del diagnóstico de la regresión se muestra en la Figura 1; en la que se aprecia en general una distribución de probabilidades entre 0 y 1 , lo que permite evidenciar un buen ajuste del modelo.

Tabla 2. Variables evaluadas en la población adulta mayor asociadas con la calidad de vida

\begin{tabular}{|c|c|c|c|c|c|c|}
\hline \multirow{2}{*}{ Variables } & \multirow{2}{*}{ Global Frec (\%) } & \multicolumn{2}{|c|}{ Calidad de vida } & \multirow{2}{*}{ OR crudo } & \multirow{2}{*}{ IC95\% } & \multirow{2}{*}{$\mathrm{p}^{*}$} \\
\hline & & Mala Frec (\%) & Buena Frec (\%) & & & \\
\hline Total & $221(100)$ & $113(51,1)$ & $108(48,9)$ & & & \\
\hline \multicolumn{7}{|l|}{ Labor } \\
\hline No & $78(35,3)$ & $53(67,9)$ & $25(32,1)$ & 2,9 & $(1,6 ; 5,2)$ & $<0,0001$ \\
\hline Sí & $143(64,7)$ & $60(42)$ & $83(58)$ & & & \\
\hline \multicolumn{7}{|c|}{ Vinculación institucional } \\
\hline Interno & $180(81,5)$ & $101(56,1)$ & $79(43,9)$ & 3,1 & $(1,5 ; 6,4)$ & 0,003 \\
\hline Externo & $41(18,5)$ & $12(24,3)$ & $29(70,7)$ & & & \\
\hline \multicolumn{7}{|l|}{ Redes sociales } \\
\hline Inadecuadas & $213(96,4)$ & $112(52,6)$ & $101(47,4)$ & 7,8 & $(0,9 ; 64,2)$ & $0,057^{* *}$ \\
\hline Adecuadas & $8(3,6)$ & $1(12,5)$ & $7(87,5)$ & & & \\
\hline \multicolumn{7}{|l|}{ CESD } \\
\hline Depresión & $124(56,1)$ & $77(62,1)$ & $47(37,9)$ & 2,8 & $(1,6 ; 4,8)$ & $<0,001$ \\
\hline Sin depresión & $97(43,9)$ & $36(37,1)$ & $61(62,9)$ & & & \\
\hline \multicolumn{7}{|c|}{ Capacidad funcional } \\
\hline Deteriorada & $126(57)$ & $75(59,5)$ & $51(40,5)$ & 2,2 & $(1,3 ; 3,8)$ & 0,004 \\
\hline Normal & $95(43)$ & $38(40)$ & $57(60)$ & & & \\
\hline \multicolumn{7}{|l|}{ Condición oral } \\
\hline Mala & 159(72) & $76(47,8)$ & $83(52,2)$ & 0,6 & $(0,3 ; 1,1)$ & 0,114 \\
\hline Buena & $62(28)$ & $37(59,7)$ & $25(40,3)$ & & & \\
\hline
\end{tabular}

${ }^{\star}$ Test de Chi cuadrado. ${ }^{\star}$ Test exacto de Fisher. 
Tabla 3. Modelo de regresión logística ajustado de la calidad de vida y la corrección de los respectivos riesgos relativos

\begin{tabular}{lcccc}
\hline \multicolumn{1}{c}{ Variable } & OR $^{*}$ & IC95\% & RR $^{* *}$ & IC95\% \\
\hline Vinculación institucional interno & 2,8 & 1,$3 ; 6,1$ & 1,6 & 1,$1 ; 1,8$ \\
No realizar labor & 2,2 & 1,$2 ; 4,2$ & 1,6 & 1,$1 ; 2,1$ \\
Depresión & 2,3 & 1,$3 ; 4,2$ & 1,6 & 1,$1 ; 1,9$ \\
\hline
\end{tabular}

${ }^{*}$ Odds Ratio ${ }^{* *}$ Razón de prevalencia.

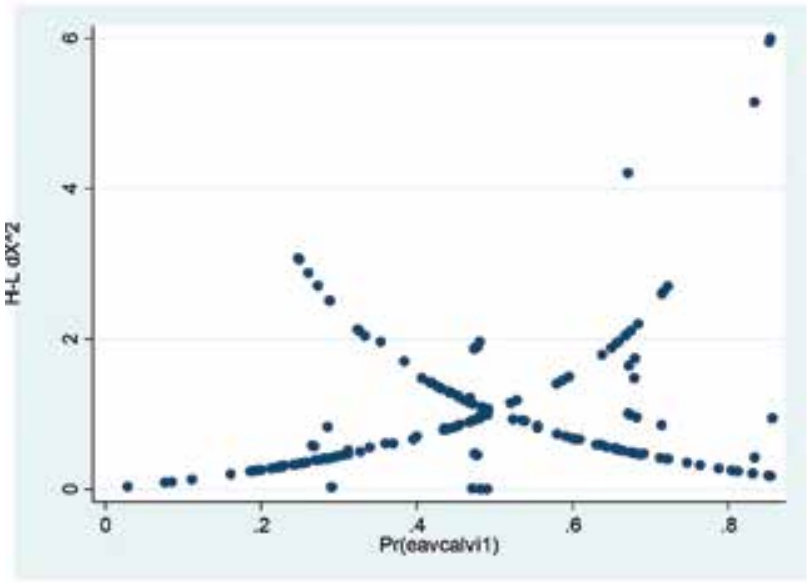

Figura 1. Diagrama de $\mathrm{dx}^{2}$ vs. Probabilidad logística estimada para el modelo de calidad de vida.

\section{DISCUSIÓN}

La calidad de vida es un objetivo en salud que está adquiriendo cada vez mayor importancia y se utiliza como medida de resultado, especialmente en situaciones en las que las actuaciones de los profesionales en esta área no solo están orientadas a prolongar la duración de la vida; sino también, que la vida que se viva sea percibida por las personas como valiosa, significativa y agradable. Esto tiene particular relevancia en los enfermos terminales y en las personas mayores ${ }^{16}$.

El presente trabajo implicó un proceso de recolección de la información y presentación de resultados en tres fases, es importante dar una visión del comportamiento de la variable calidad de vida, analizada a lo largo del proceso desarrollado. En la etapa inicial de este trabajo ejecutada en una institución geriátrica, se evidenció que el puntaje alcanzado para calidad de vida obtuvo una media de $68,7 \pm 19,9$, mediana de 70,5 y rangos entre 10 y 100 puntos. En la segunda fase del proceso, analizado en tres hogares, el promedio de calidad de vida reportado por las personas mayores fue de
$49,9 \pm 18,6$, mediana de 52 y rangos entre 14 y 85 años ${ }^{1}$. En esta la tercera y última fase en las que se incluyeron 6 instituciones geriátricas, la media de calidad alcanzó un puntaje de 55,8 $\pm 20,4$ con mediana de 58 y rangos entre 12 y 100 puntos. Estos resultados permiten evidenciar la variabilidad de la percepción sobre calidad de vida que pueden registrar las personas mayores y que es producto de la subjetividad, aspecto inherente al concepto que sobre calidad de vida existe ${ }^{16}$.

En una población mexicana de adultos mayores, Jiménez-Jérez y colaboradores evaluaron la calidad de vida, encontrando que el $11 \%$ de estas personas registró esta condición comprometida y el $84 \%$ de estos registró puntajes entre 80 y 100. En el presente trabajo el $54,3 \%$ registró mala calidad de vida y el $48,9 \%$ registró puntajes superiores a 58; con lo que se evidencia una peor percepción en la condición de vida en los mayores de Bucaramanga. Las diferencias evidenciadas podrían sustentarse en el hecho de que la población mexicana evaluada residía en sus propios hogares, mientras que la de nuestra ciudad estaba recluida en instituciones, y registraba, además, compromiso físico, mental y redes sociales frágiles, lo que compromete su percepción de calidad de vida ${ }^{17}$.

Las personas mayores institucionalizadas de Bucaramanga evaluadas en el presente trabajo registraron su condición bucal comprometida, aspecto que podría catalogarse como un problema de salud pública por el cúmulo de eventos bucodentales que registran, lo que indica la necesidad de promover el acceso a la atención de este colectivo de adultos mayores; este acceso deberá incluir acciones promocionales soportadas en la educación para la salud, pero también de prevención primaria, secundaria y terciaria, contrarrestando con esto los factores de riesgo y los determinantes sociales de la salud bucal que estos adultos mayores institucionalizados enfrentan ${ }^{18}$. El estado de salud bucal deficiente que se observa en las personas mayores, aunado a las proyecciones que sobre necesidad de atención se 
plantean para este grupo, revelan la urgente necesidad de fortalecer los programas de gerodontología que a nivel local, regional, nacional y latinoamericano se desarrollan, en los que se hace necesario aunar esfuerzos y promover el trabajo en red orientados a fortalecer el intercambio de experiencias.

Analizar la salud y la calidad de vida en las personas mayores implica comprender su salud física, su capacidad funcional, sus condiciones sicológicas, mentales y sociales, por la estrecha relación que existe entre estos diferentes componentes de la salud integral. Considerando esto, es importante señalar que los cambios físicos que se registran con el envejecimiento como es el aumento de la tensión arterial, en los niveles de glicemia, las alteraciones en las funciones, digestivas, renales y hepáticas, entre otras, predisponen el deterioro funcional y mental, lo que incide sobre la calidad de vida en las personas mayores ${ }^{19,20,21}$. Estos aspectos parecen evidenciarse en la población adulta mayor vinculada a las seis instituciones geriátricas analizadas, en las que se observó que el 77,8\% de las personas mayores recibía algún tipo de medicación, tenían en promedio 2,8 sistemas comprometidos y una media de consumo de medicamentos cercana a 2. A pesar de mostrar la condición general deteriorada en los adultos mayores evaluados en Bucaramanga, Locker y \& Gibson reportaron un mayor deterioro en una población mayor en Canadá, en la que encontraron que en promedio registraban 4,5 sistemas comprometidos y cerca del $93 \%$ se les administraba más de un medicamento; cabe señalar, la población canadiense registraba una media de edad de 83,3 años en tanto que la población de mayores de Bucaramanga registró una media de 75 años; por lo que se podría conjeturar que las diferencias en la condición sistémica podrían relacionarse a incremento de la edad ${ }^{22}$.

La salud mental abarca factores relacionados con el desarrollo, la depresión, la demencia senil y la disminución del funcionamiento cognitivo, ya que como menciona la OMS son las que más se presentan en las personas mayores. Las investigaciones realizadas, específicamente en depresión, plantea que esta se relaciona con la continua hospitalización y la permanencia en hogares geriátricos que lleva al aumento de la mortalidad y morbilidad, afectando finalmente la percepción de salud y los cuidados que se tengan ${ }^{23}$.
Espíndola y colaboradores en un estudio realizado en Bucaramanga en el 2003 mostró que el 56,3\% de la población adulta mayor institucionalizada registró depresión, cifra similar a la reportada en la presente investigación $(56,1 \%)$ lo que parece indicar que esta condición aqueja a los adultos mayores de Bucaramanga y la necesidad de intervenciones médicas, psicológicas y de terapistas ocupacionales que les ayuden a manejar las condiciones mentales que enfrentan al institucionalizarse ${ }^{24}$.

Gómez \& $\mathrm{Cursio}^{25}$ en un estudio que evaluó el deterioro físico y funcional en ancianos en Manizales, mediante la escala de Barthel, encontraron que el $35,2 \%$ de las personas mayores tenían algún nivel de dependencia. En la población adulta mayor de Bucaramanga en este trabajo el deterioro físico se evidenció en el $57 \%$ de las personas, lo que parecería indicar que el deterioro funcional fue mayor en estos últimos; aunque la diferencia en los índices utilizados podría explicar estas discrepancias. En todo caso, el deterioro físico es una condición que no puede subestimarse en los mayores por sus implicaciones sobre la vida y la calidad de vida en los grupos sociales, y afectar la realización de actividades básicas para la vida como son alimentarse, hacer el aseo personal, vestirse y desplazarse ${ }^{25}$. La relación entre la discapacidad física con la calidad de vida ha sido estudiada, encontrando, por ejemplo, que el $60 \%$ de las personas que presentaban discapacidades físicas consideraban que tenían comprometida su calidad de vida, cifras similares a las encontradas en el presente estudio en el que el 59,5\% de las personas con capacidad física deteriorada registraban tener mala calidad de vida ${ }^{1,18}$.

$\mathrm{Al}$ analizar las variables que en este trabajo se asociaron positivamente con calidad de vida cabe resaltar la vinculación institucional como interno (OR 3,1), la falta de realización de labores (OR 2,9), las redes sociales inadecuadas (OR 7,8), la depresión (OR 2,8), el deterioro funcional (OR 2,2); resultados similares se evidenciaron en el trabajo de Rodríguez Feijóo ${ }^{26}$ : "Factores que influyen sobre la calidad de vida de los ancianos que viven en geriátricos", pues concluye que el nivel educacional, el uso del tiempo libre y el apoyo social influyen significativamente sobre la calidad de vida del anciano institucionalizado. 
La calidad de vida en las personas mayores se relaciona con el estado de salud, pero también con la independencia física y económica por su incidencia sobre la autonomía de las personas. El hecho que, las personas mayores residan en hogares geriátricos, compromete la independencia del anciano, porque al estar institucionalizado la persona mayor dependerá de su relación con sus cuidadores, con los que probablemente compartan muy pocas cosas en común. En este sentido, independiente de la institucionalización, el apoyo de los familiares y amigos de la persona mayor se convierte en un elemento crucial para que perciba que cuenta con redes de acompañamiento de apoyo, colaboración y de amor filial que impactan enormemente en la autoestima del anciano.

Siempre que se pueda, el adulto mayor debe vivir en su propio hogar, recibiendo el apoyo afectivo que merecen, por supuesto contando con el acompañamiento y la ayuda que requiera, pero promoviendo su independencia; solo deben internarse cuando, sus condiciones de fragilidad física, de carencia económi$\mathrm{ca}$, de redes sociales o dependencia funcional lo determinen y esta es quizás las condiciones que enfrentan los adultos mayores evaluados en esta investigación ${ }^{26}$.

Rojas y $\mathrm{Col}^{27}$ por su parte afirman que los centros de atención residencial para personas mayores son necesarios, pues son una respuesta a los problemas de las personas en edad avanzada que viven solas, no tienen soporte familiar, o porque deciden vivir en este entorno; sin embargo, estos autores expresan igual que Rodríguez Feijóo que la institucionalización debería ser el último recurso, ya que se identifica como uno de los factores que actúan en la disminución de la autonomía de la persona mayor ${ }^{26,27}$.

El adulto mayor se ve obligado a compartir su vida con personas desconocidas, habituarse a horarios, hábitos y dietas extrañas, por lo cual tienen mayor riesgo a enfermar. Los ancianos sufren lo que se denomina "las pérdidas asociadas a su envejecimiento" pues sienten pérdidas en un entorno al que no dominan, se destruyen las relaciones que han tenido a lo largo de su vida; la institucionalización es un motivo de vulnerabilidad para el anciano. La persona pierde la capacidad para lograr retener el control de su propia vida y la capacidad de decidir sobre cualquier aspecto de su vida cotidiana; pues lo lleva a "dejar de hacer" todo con el propósito de adaptarse al medio, pero esta práctica actúa negativamente a mediano y largo plazo en la propia percepción de salud que tiene la persona mayor ${ }^{27}$.

Es fundamental considerar además las potenciales dificultades que pueden surgir en la administración de las instituciones geriátricas; pues en aras de la organización, se hace necesario determinar para estas, normas que usualmente se establecen en forma rígida, que obedecen sistemas de organización que limitan el contacto de los ancianos con el mundo exterior dentro de un sistema más regulado, formalizado e impersonal; en el que el anciano no puede tomar decisiones y llevar a cabo tareas cotidianas; se produce además el "stress por reubicación" y en el que el ingreso a una residencia implica desarraigo y sobreesfuerzo de adaptación. Por lo cual la internación de la persona mayor trae consigo un aumento en su grado de dependencia y un deterioro más acelerado de sus condiciones físicas y síquicas ${ }^{26}$.

Se destaca además, que estudios ilustran cómo la relación con otros internos y con el personal de la institución es con frecuencia insatisfactoria y las personas mayores reportan estar descontentas con el apoyo familiar que reciben una vez son institucionalizadas. Lo expuesto parece explicar por qué internarse en un hogar geriátrico se constituye en un factor que puede asociarse con una mala calidad de vida y vulnerar su salud física y mental ${ }^{26}$. Considerando lo anterior, se hace necesario que la institución ofrezca a la persona que debe ser recluida, diferentes alternativas de actividades para que el adulto mayor pueda realizar aquellas que le resulten más interesantes y atractivas, aumentando así su sensación de autoeficacia y evitando que el comportamiento del anciano sea pasivo, fenómeno que es bastante frecuente en las personas mayores institucionalizadas.

Las directivas de los centros geriátricos deben informar y buscar el apoyo de los familiares; deben también, señalar la importancia que tiene para el anciano contar con su apoyo, recibir visitas, sentir que su familia sigue siendo su grupo de pertenencia, su red de apoyo para disminuir de este modo los sentimientos de soledad, desarraigo y abandono, contribuyendo de este modo a mejorar su calidad de vida y a reducir los sentimientos de depresión y de tristeza ${ }^{26}$. 
Cuando se hace alusión a la relación de falta de labores con la calidad de vida, se debe considerar lo expuesto por Mingorance, quien afirma el desarrollo de actividades de tipo sociocultural en el tiempo libre de los ancianos puede ser un elemento que influye sobre el bienestar de los sujetos; considerando esto, se hace necesario mejorar las aptitudes y la actitudes para ayudarle al adulto mayor a desenvolverse en la vida diaria, cultivar relaciones interpersonales, participar y contribuir en el desarrollo y cambio social, permitiendo a las personas ser independientes y autónomas, descubrir el sentido de su vida, siendo protagonistas en la construcción de su vida. Por lo cual se hace necesario convertir el tiempo libre del que disponen los ancianos, en un espacio de formación integral que consigue reducir los achaques relacionados con la edad, potenciar la alegría y la actividad dinámica, luchar contra el sedentarismo y la apatía, desterrar la soledad y la tristeza aumentando la autoestima y teniendo presente que "el verdadero protagonista de la vida es cada persona" 28 .

Es importante clarificar sobre las posibles limitaciones de este trabajo, y tiene que ver con la realización de esta investigación soportada en fuentes secundarias a partir de una investigación que se proponía evaluar la asociación de la calidad de vida con la condición oral, por lo que el poder de este estudio para las condiciones físicas, mentales y sociales evaluadas podría estar comprometido; con todo el modelo generado permitió evidenciar aspectos cruciales como estar internos, sin una actividad laboral y con depresión como criterios que inciden sobre la calidad de vida . La valoración integral se podría señalar como una fortaleza de este trabajo y ratifican la necesidad de una visión integral e integradora de las intervenciones que sobre los adultos mayores institucionalizados deben realizarse para incentivar con ello su calidad de vida1; además, la implementación de índices e instrumentos validados sobre población adulta mayor aportan valor metodológico al proceso investigativo realizado ${ }^{6}$.

Se concluye que estar interno en una institución geriátrica, no realizar ninguna labor y sufrir de depresión son los predictores de la mala calidad de vida en las personas mayores residentes en los hogares geriátricos evaluados de la ciudad de Bucaramanga.

\section{Agradecimientos}

Se agradece a la Universidad Santo Tomás Seccional Bucaramanga por el apoyo financiero para la realización de esta investigación.

\section{Conflicto de interés}

No existe ningún conflicto de interés en la realización de este trabajo

\section{REFERENCIAS}

1. Concha SC, Camargo DM. Análisis de la asociación entre la calidad de vida y la condición oral de las personas mayores vinculadas a tres instituciones geriátricas de Bucaramanga: II Fase. UstaSalud. 2007;6(2):75-86.

2. Huenchuan S. Envejecimiento, derechos humanos y políticas públicas. ONU, CEPAL, CELADE, Santiago de Chile, 2009. (Citado en marzo de 2014). Recuperado a partir de: http://social.un.org/ageing-working-group/documents/ECLAC_sp_HR\%20and\%20public\%20policies. pdf

3. Sardi E. Cambios sociodemográficos en Colombia: Periodo intercensal 1993-2005. Revista de la Información Básica; 2(2). (Citado en marzo de 2014). Recuperado a partir de http://www.dane.gov.co/revista_ib/html_r4/ articulo2_r4.htm

4. Ruiz NY. El desplazamiento forzado en el interior de Colombia: Caracterización sociodemográfica y pautas de distribución territorial 2000-2004. [Tesis doctoral]. España: Universidad Autónoma de Barcelona; 2007. (Consultado en marzo de 2014).

5. Goldberg TH, Chavin SI. Preventive Medicine and Screening in Older Adults. J Am Geriatr Soc. 1997;45(3):34454. doi: 10.1111/j.1532-5415.1997.tb00952.x.

6. Wallance RB, Woolson RF. The epidemiologic study of elderly. Oxford: University press. 1992.

7. Fried LP. Epidemiology of Ageing. Epidemiologic Reviews. 2000;22(1):95-106.

8. Banco Mundial. Desarrollo sostenible en un mundo dinámico, transformación de instituciones, crecimiento y calidad de vida. Informe sobre el desarrollo mundial. Bogotá: Alfaomega Editores, 2003.

9. Restrepo H, Málaga H. Promoción de la salud: Como construir vida saludable. Bogotá: Editorial Médica Panamericana, 2002. 
10. Lugo LH, García HI, y Gómez C. Calidad de vida y calidad de vida relacionada con salud. IATREIA. 2002,15(2):96-102.

11. Buendía J. Gerontología y salud. Perspectivas actuales. Madrid: Editorial Biblioteca Nueva; 1997.

12. Torres IA, Beltrán FJ, Saldivar AH, Ochoa DL, Barrientos MC, Pérez H, Navarro AM. La salud mental, una aproximación a su estudio en poblaciones vulnerables. Revista Electrónica Medicina, Salud y Sociedad. 2013;3(2):1-33. doi:10.25009/remsys.2013.2.103.

13. Botero BE, Pico ME. Calidad de vida relacionada con la salud en adultos mayores de 60 años: una aproximación teórica. Revista hacia la Promoción de la Salud; 2007;12:11-24.

14. Organización Mundial de la Salud. Promoción de la salud mental. Conceptos, evidencia emergente, práctica. Informe compendiado, 2004. (Citado en febrero de 2012). Recuperado a partir de: http://www.who.int/mental_health/ evidence/promocion_de_la_salud_mental.pdf

15. Zhang J, Yu KF. What's the relative risk? A method of correcting the odds ratio in cohort studies of common outcomes. JAMA. 1998;280(19):1690-1. doi:10.1001/ jama.280.19.1690.

16. Azpiazu M, Cruz JA, Villagrasa JR, Abanades JC, García N, Álvarez C. Calidad de vida en mayores de 65 años no institucionalizados de dos áreas sanitarias de Madrid. Aten Primaria. 2003;31(5):285-94. doi: 10.1016/ S0212-6567(03)79180-X.

17. Jiménez J. Esquivel I, González-Celis AL. La percepción de la salud bucal como media de calidad de vida en ancianos. Rev. ADM. 2003;10(1):19-24.

18. Orozco MV, Orozco LC, Herrera JP, Méndez G, Mendoza MY, Pinzón LI, y Sánchez VJ. Validación de un instrumento para medir la calidad de vida en sus aspectos físicos, emocional, cognitivo y social en personas ancianas. Rev. Asoc Colomb. Gerontol. Geriatric. 2004;18(1-2):606-12.
19. Brenes W, Hoffmaister F. Situación de salud bucal en personas adultas mayores: problemas y desafíos. Rev costarric. cienc. Méd. 2004;25:(3-4): 27-41.

20. Anzola PE, Morales MF. La atención de los ancianos: Un desafío para los años noventa. Organización Panamericana de la Salud. Publicación Científica. 1994;546:3-435.

21. Shimazaky Y. Soh I. Saito T. Yamashita Y. Koga T. Miyazaky H. Takehara T. Influence of dentitions status on physical disability, mental impairment, and mortality in institutionalized elderly people. In: J. Dent Res. 2001;80(1):340-345. doi:10.1177/00220345010800010801.

22. Locker D. Gibson B. Discrepancies between self-ratings of and satisfaction with oral health in two older adult populations. Community Dent Oral Epidemiol. 2005;33(4):280288. doi:10.1111/j.1600-0528.2005.00209.x.

23. Uribe AF, Valderrama LJ, Molina Linde JM. Salud objetiva y salud psíquica en adultos mayores colombianos. Acta Colombiana de Psicología. 2007;10(1):75-81.

24. Espindola G. Pinzón AR, Daza CR. Concha SC y Camargo DM. Asociación entre el estado mental y la condición oral de la persona adulta mayor institucionalizada de la ciudad de Bucaramanga y su área metropolitana. UstaSalud. 2003;2(1):31-32.

25. Gómez JF, Cursio CL. Autopercepción de salud, presencia de enfermedades y discapacidades en ancianos de Manizales.Rev. Asoc. Colomb. Geriatric. 2004;18(4):706-714.

26. Rodríguez N. Factores que influyen sobre la calidad de vida de los ancianos que viven en Geriátricos. Psicología y Psicopedagogía. 2007;6(17):1-8.

27. Rojas MJ, Toronto A, Rodríguez Ponce C, Rodríguez JB. Autonomía y estado de salud percibidos en ancianos institucionalizados. Gerokomos. 2006;17(1):6-23.

28. Mignorance AC. El tiempo libre de las personas mayores a través de la animación sociocultural representada como una "educación formal no escolarizada". Revista Iberoamericana de Educación. 2008;45(6):1-12. 